Research Article

\title{
From Graphite to Graphene: The Confinement of the Fermi Surface to the Line KH
}

\author{
Helmut Bross \\ Arnold Sommerfeld Center, University of Munich, Theresienstraße 37 IV, 80333 Munich, Germany \\ Correspondence should be addressed to Helmut Bross; helmut.bross@theorie.physik.uni-muenchen.de
}

Received 7 March 2013; Accepted 9 April 2013

Academic Editors: K. Kasamatsu, A. N. Kocharian, V. Kochereshko, R. Rossmanith, and S. Wang

Copyright (C) 2013 Helmut Bross. This is an open access article distributed under the Creative Commons Attribution License, which permits unrestricted use, distribution, and reproduction in any medium, provided the original work is properly cited.

The graphite structure is self-consistently calculated by use of the all electron Modified Augmented Plane Wave (MAPW) scheme with lattice constants $c$ considerably enlarged above the experimental value of graphite. Overall, the band structures of the series are found to be quite similar: the energy levels of the highly symmetric states $\mathrm{K}$ and $\mathrm{H}$ almost coincide, essentially fixing the Fermi level of the semimetallic solid. The dispersion along lines parallel to the atomic planes, already small in graphite at the experimental value of $c$, continues to flatten with increasing value of $c$. The structure with an interlayer distance enlarged by the factor 3 over the experimental value provides a good approximation of the behaviour of a monoatomic sheet. In this context, the unusual behaviour of graphene appears in a new light.

\section{Introduction}

Because of its layered structure with relatively large separation between the layers, graphite has been modelled as a two-dimensional solid in the first theoretical investigations based on various methods [1]. In the following decade, the full three-dimensional crystal structure has been investigated by all modern band structure schemes: pseudo-potential [2-5], full-potential linearised augmented plane wave [6, 7], full-potential linear muffin-tin-orbital [8], full-potential linear combination of Gaussian-type orbitals fitting-function technique (LCGTO-FF) [9] which all, due to inherent systematic restrictions, produce different degrees of accuracy. To understand the extraordinary behaviour of graphene we use the reverse strategy and study how the electronic structure of the graphite structure changes with increasing interlayer distance. We find that the semimetallic behaviour is drastically reduced as the Fermi surface shrinks to the surrounding of the $\mathrm{KH}$ line accompanied with a loss of the dispersion in the relevant bands. Only highly accurate schemes can properly describe this phenomenon. By a proper choice of the intrinsic parameters and with some specific care the MAPW method $[10,11]$ satisfies this criterion. The present investigations raise doubts on previous investigations of graphite and graphene based on the tight binding scheme with the nearest and second nearest neighbours couplings [12-14].

The outline of the paper is as follows: in Section 2 some issues of the crystallography of graphite and graphene are described. Then we sketch some features and the proper choice of intrinsic parameters of MAPW as well as further details of the calculation. Section 3 reports the results of self-consistent calculations done with increasing interlayer distance. Especially, we discuss some common features in the band structure. Then we describe in Section 4 the strategies to deal with the semimetallic behaviour. The construction of the Fermi surface (FS) clearly illustrates the confinement in $\vec{k}$ space, which has far reaching consequences. Finally, Section 5 gives a short outlook on other electronic features of graphite and graphene. Unfortunately, a detailed comparison with experiments is not possible since their results are mostly discussed in context of the Slonczewski-Weiss-McClure [13, 14] model.

\section{Electronic Structure}

2.1. Crystallography of Graphite and Graphene. The Bravais lattice of graphite is primitive hexagonal with the basis $\vec{a}_{1}=$ $(0,-1,0) a, \vec{a}_{2}=(\sqrt{3} / 2,1 / 2,0) a, \vec{a}_{3}=(0,0,1) c$ [15]. Its crystalline structure consists of equidistant planes of carbon 
atoms, each forming a hexagonal net, stacked in a manner such that half of the carbon atoms (type A) are located directly above each other in adjacent planes, while the other half (type B) are located above the centres of the hexagons in the adjacent plane [1]. There are four atoms per unit cell. The space group of graphite is $P 6_{3} / \mathrm{mmc}=D_{6 h}^{4}$, No. 194 [15]. The corresponding point group has 24 elements and is isomorphic to $D_{6 h}$. Half of the operations of the point group have nonprimitive translations $\vec{v}=\vec{a}_{3} / 2$ associated with them, carrying from an atom on one plane to an atom of the same type on the adjacent plane.

The numerical work is considerably reduced if we choose the origin to be located halfway between adjacent layers on a $c$-axis through atoms of type A. It is an inversion centre of the point group with the advantage that the Fourier transforms of the charge density, the crystal potential, and other quantities invariant under the operations of the space group are realvalued. In this choice of the elementary cell the atoms of the basis are located at

$$
\begin{array}{ll}
\text { A atoms: } & \vec{R}^{1}=\frac{\vec{a}_{3}}{4}, \\
\text { B atoms: } & \vec{R}^{2}=\frac{\left(\vec{a}_{1}+2 \vec{a}_{2}\right)}{3}+\frac{\vec{a}_{3}}{4}
\end{array}
$$

and two further atoms in inversion positions.

In graphene we have only the basis vectors $\vec{a}_{1}$ and $\vec{a}_{2}$. The pattern of carbon atoms looks the same as that of one plane in graphite. But there exist no crystallographic reasons to distinguish closely neighboured atoms on a hexagon ring. Again its 2-dimensional space group is composed of the point group $D_{6 h}$ where the nonprimitive translation $\vec{v}=\left(\vec{a}_{1}+\right.$ $\left.2 \vec{a}_{2}\right) / 3$ is associated with half of its operations.

2.2. Some Details of the Band Structure Calculations. The electronic structure was self-consistently determined by use of the modified augmented plane-wave (MAPW) formalism [10]. Common to most modern schemes, the constituents within the atomic polyhedron are surrounded by nonoverlapping spheres (APW spheres). Outside these spheres the Bloch functions $\langle\vec{r} \mid n \vec{k}\rangle$ are approximated by superposition of plane waves, whereas within each APW sphere the plane waves are suitably augmented to properly describe the atomic character of the wave function. Specifically, in the MAPW scheme, the angular decomposition of $\langle\vec{r} \mid n \vec{k}\rangle$ is a sum of products of spherical harmonics $Y_{l m}$ and a set of radial functions $R_{s l}$ where $s$ is associated with various orbital energies. The $R_{s l}$ are generated in a spherical averaged potential, mostly by requiring that their logarithmic derivative $d R_{s l} / d r$ is either 1 or -1 . To avoid any truncation error, the augmentation of the plane waves is only performed for the leading angular momenta $l \leq l_{\max }$, whereas for $l>l_{\max }$ the spherical Bessel functions $j_{l}$ are kept. In contrast to all other versions of the APW scheme [6], the full-wave function and its derivative are made exactly continuous on the surface of the APW spheres by use of additional constraints in the extremal problem, with the consequence that the corresponding eigenvalue problem is a generalised one, but is real symmetric.
Test investigations in the full atomic configuration $1 \mathrm{~s}^{2}$, $2 s^{2}, 2 p^{2}$ of $C$ had the results that the two lowest bands are well separated from the other bands by more than $18 \mathrm{Ry}$ and do not significantly influence the valence electrons. Thus the 1s electrons are considered to constitute the core which, however, is not kept fixed in the self-consistent cycles. Thanks to the strong localisation of the core wave functions the valence states are almost fully orthogonal on the core states. Due to the strong s-p character of graphite we choose $l_{\max }=1$ and a set of radial functions $R_{s l}$ consisting of 4 elements for each $l=0,1$ with alternating sign of the logarithmic derivative.

In the present study which focuses on the influence of increasing interlayer distance the choice of the plane waves used to describe a Bloch state $\vec{k}$ is rather subtle. We have found that the inequality

$$
\left(\vec{k}_{\|}+\vec{K}_{\|}\right)^{2}+\left(k_{\perp}+K_{\perp}\right)^{2} \leq \mathrm{QMXSQ}
$$

which limits for a given value of QMXSQ the kinetic energy of the plane waves and guarantees that the correct symmetry behaviour in $\vec{k}$ space is most appropriate. $\|$ denotes the components of $\vec{k}$ and $\vec{K}$ parallel to the graphite sheets and $\perp$ their perpendicular components. This requirement has the effect that the wave functions in the interplanar space are properly described as the number of reciprocal vectors $\vec{K}$ increasing due to their perpendicular components when the interlayer distance grows. For any value of the wave vector $\vec{k}$ the actual value of QMXSQ fixes the rank of the MAPW eigenvalue problem and thus controls the accuracy of the Bloch eigenvalues $E_{n \vec{k}}$. Cursory investigations have been done with the value QMXSQ $=20.16$ a.u. corresponding to a maximal kinetic energy of the same value in Rydberg.

The density of the electrons $\rho(\vec{r})$ obtained from the occupied states is, as a consequence of the MAPW ansatz, a symmetric combination of plane waves everywhere, superimposed by an angular-dependent contributions that are treated in the accuracy wanted by use of a suitably chosen fine mesh of $\vec{r}$ points [16].

The Brillouin zone (BZ) integrations over all occupied states yielding the electron density $\rho(\vec{r})$ and the total energy $E_{\text {tot }}$ are approximated by a sum over $\vec{k}$ points properly chosen in the irreducible wedge with the concept of Monkhorst and Pack [17] applied to the hcp structure. They are part of a hexagonal Bravais lattice spanned by the basis vectors $\left(\pi / \mathrm{MG}_{1} a, \pi / \mathrm{MG}_{2} a, \pi / \mathrm{MG}_{3} c\right)$ which avoids locating the points $\mathrm{H}$ and $\mathrm{K}$ in the centre of the parallelepipeds. Here, the $\mathrm{MG}_{i}, i=1,2,3$ are specially chosen integers. Thus the influence of possible energy degeneracies is kept small. Within the self-consistent cycles the same value of QMXSQ is used which has been chosen to keep the fluctuation of the rank of the eigenvalue problems small.

The characteristic ground-state properties are calculated within the local-density approximation (LDA) by use of the exchange-correlation functionals $[18,19]$. As we found no significant difference we restrict ourselves in the following to results obtained with the functional [19]. 


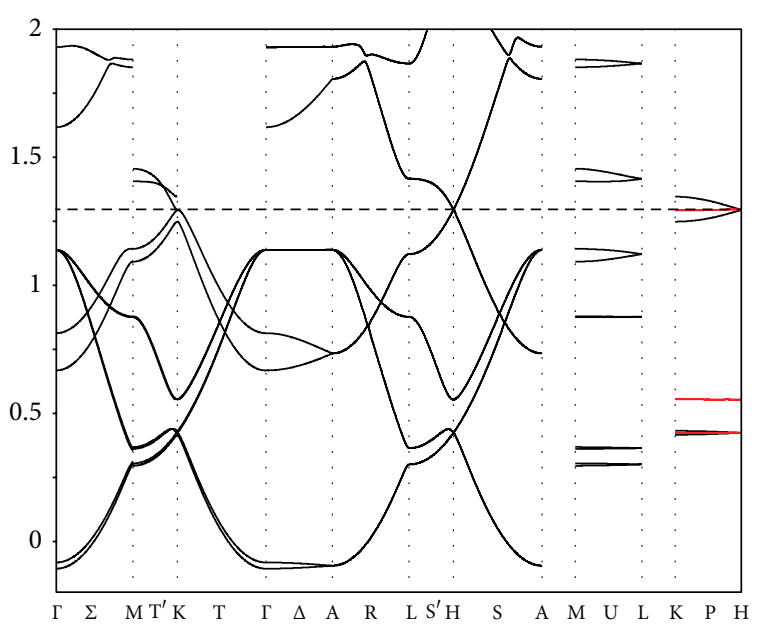

FIgURE 1: Band structure along different lines in the Brillouin zone for the value $c=12.6536$ Bohr. The notation of Bradley-Cracknell [15] is used. The broken line shows the Fermi level. In the outermost panel the twofold degenerated bands are marked red. All energies are in Ry.

\section{Results: Some Common Features of the Band Structure}

Self-consistent calculations were performed for the lattice constant $a=2.456 \AA=4.641167$ Bohr [1] and the lattice constant $c$ varies from $6.696 \AA=12.653605$ Bohr [1] up to 36.0 Bohr.

Figure 1 shows the band structure along different special directions of the Brillouin zone characteristic for the experimental value of $c$. Up to $c=36$ Bohr the band structures look quite similar to previous investigations apart from a rigid shift caused by the Ewald potential and a global compression by $5.9 \%$ against the LCGTO-FF calculations [9] in the energy scale.

(1) The low lying bands are well separated from the excited states. Both groups are only connected at the special points $\mathrm{H}$ and $\mathrm{K}$ which have almost coinciding energies. Table 1 lists the values of these energies for different values of the lattice parameter $c$. With increasing value of $c$ the energies $E_{\mathrm{K}}$ and $E_{\mathrm{H}}$ next to the Fermi energy get closer without any order in the sign. The low lying bands completely accommodate the $2 \mathrm{~s}$ and $2 \mathrm{p}$ electrons of graphite for all values of $c$ considered. The well-separated excited states are not occupied. The Fermi level is close to the eigenvalues of the $\mathrm{H}$ and $\mathrm{K}$ states quoted in Table 1 . Its relative location to these values determines the semimetallic behaviour.

(2) Parallel to the carbon planes, for example, along the lines $\Delta, \mathrm{U}$, and $\mathrm{P}$, the dispersion of the bands weakens with increasing lattice constant $c$ and is almost negligible at $c \geq 24.0$ Bohr. To make this more clear, the four bands closest to the Fermi level $\epsilon_{F}$ are displayed in Figure 2 for different values of $c$ on an enlarged scale. Both outermost curves mark nondegenerate states, whereas the curve inbetween mark's two-fold degenerate states. In previous investigations these bands were denoted by $E_{1}, E_{2}$, and $E_{3}$, respectively. The twofold degeneracy is lifted as we move away from the zone edge; one of these bands will host the Fermi level. All energies are given as offsets to the energy values of the twofold states at the point $\mathrm{K}$. Their scales have been chosen in such a way that their shapes become similar. It is worth mentioning that the scales vary by more than two order of magnitude when the interlayer distance is doubled. For the values of $c$ considered the twofold generated band is cut by the Fermi level and thus separates occupied and non occupied states. Further details are discussed in the next section.

(3) The metallicity becomes distinctly apparent in the density-of-states-curve, displayed in Figure 4, by a fine dip near the energies of the twofold generated band (red curve) which sharpens with increasing value of the lattice constant $c$. Within the accuracy of the plotting our band structure fairly well agrees with that obtained with a special version of the Gaussiantype orbitals technique (LCGTO-FF) [9]. Especially, both calculations show a splitting at the bottom of the occupied bands at the point $\Gamma$ in contrast to the FLMTO investigations [8].

\section{Strategies to Deal with the Semimetallic Behaviour}

With increasing value of the lattice constant $c$, the surface dividing occupied and nonoccupied states (Fermi surface) almost completely contracts to the line $\mathrm{KH}$. In the energy region of interest the $\mathrm{H}$ point hosts two twofold degenerate eigenstates with energies differing by $0.034 \mathrm{mRy}$, whereas in the $\mathrm{K}$ point four eigenvalues cover the range of $0.160 \mathrm{mRy}$ with a twofold degenerate state inbetween. Both values refer to $c=24.0$ Bohr with the tendency to shrink in the limit $c \rightarrow \infty$.

Because of the confinement in $\vec{k}$ space all schemes to locate the Fermi surface, originally designed for ordinary metals, fail even if the number of sampling points of the wave vector is increased. On the other hand, it allows to use the $\vec{k} \cdot \vec{p}$ approximation, which has already been applied within the initial investigations of graphite $[12,13]$, based on the four almost degenerate eigenstates on the $\mathrm{KH}$ line. For fixed value of $k_{\perp}, 0 \leq k_{\perp} \leq 2 \pi / c$, we get an eigenvalue problem of rank 4

$$
\Sigma_{j} H_{i j} x_{j}=\varepsilon x_{i},
$$

where the leading contribution to the matrix elements $H_{i j}$ originates from the components of the momentum operator $\vec{p}$. Apart from $k_{\perp}$ the elements $H_{i j}$ depend on the twodimensional vector $\vec{\kappa}$ perpendicular to the vector characterising the $\mathrm{KH}$ line, for example, $(2 \pi / a \sqrt{3}, 2 \pi / 3 a)$. As already mentioned, it is useful to define the energy eigenvalues $\varepsilon$ relative to the energy of the degenerate eigenstate on the line $\vec{\kappa}=0$. 

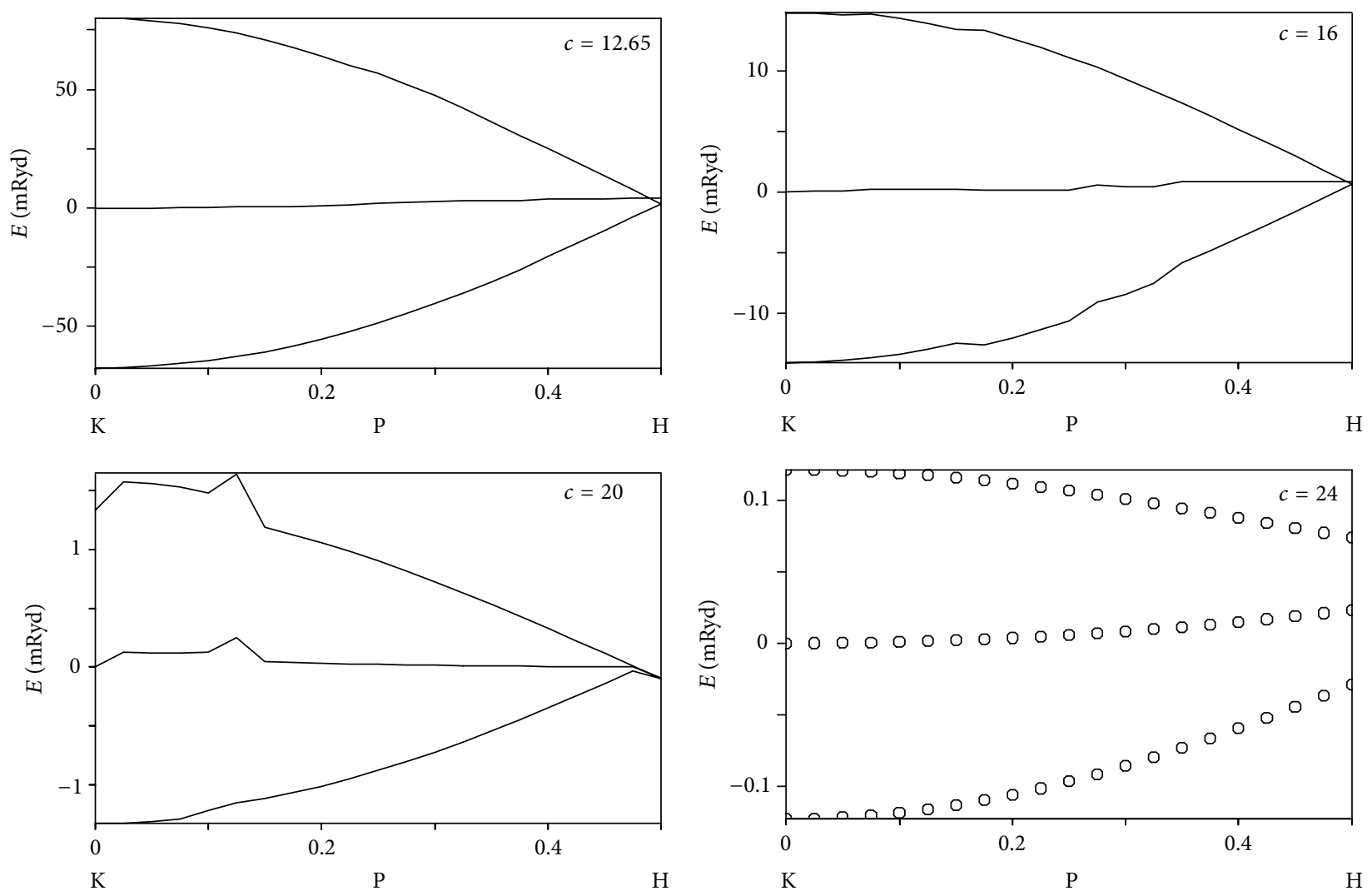

(a)

(b)

FIGURE 2: Band structure along the line KH for the experimental lattice constant $c=12.6536$ Bohr, and the enlarged values $c=16.0$ Bohr, $c=20.0 \mathrm{Bohr}$ and $c=24.0 \mathrm{Bohr}$. The middle, almost flat, curve shows the twofold degenerate band, whereas the other mark the nondegenerate bands. Horizontal axis in units of $2 \pi / c$. The notation of the relevant bands is in accord with previous work. Note the close agreement in the case of $c=12.6536$ Bohr with the result displayed in Figure 7(a) [20] or Figure 2 [5] obtained by LDA and GW calculations.

TABLE 1: The energy eigenvalues of the point $\mathrm{K}$ and the eigenvalues of the twofold degenerated state $\mathrm{K}$ in units of Rydberg with increasing interlayer distance. For each value of $c$, by proper choice of QMXSQ, the set of the plane waves is generated which minimises the fluctuations of the number of plane waves NPW along the line KH.

\begin{tabular}{lcccc}
\hline$c($ Bohr $)$ & NPW & $E_{\mathrm{K}}$ & $\mathrm{NPW}$ & $E_{\mathrm{H}}-E_{\mathrm{K}}$ \\
\hline 12.6536 & 339 & 1.295390 & 336 & 0.002543 \\
14.0 & 378 & 1.165958 & 378 & 0.000442 \\
16.0 & 441 & 1.004455 & 438 & 0.000085 \\
18.0 & 483 & 0.872945 & 483 & 0.000449 \\
20.0 & 537 & 0.762965 & 540 & -0.000219 \\
22.0 & 615 & 0.669224 & 612 & 0.000198 \\
24.0 & 669 & 0.589686 & 666 & -0.000064 \\
26.0 & 708 & 0.521203 & 708 & 0.000010 \\
28.0 & 771 & 0.461227 & 768 & 0.000020 \\
30.0 & 813 & 0.408885 & 816 & 0.000233 \\
32.0 & 885 & 0.362247 & 882 & 0.000189 \\
34.0 & 921 & 0.321406 & 918 & 0.000167 \\
36.0 & 981 & 0.284721 & 978 & 0.000060 \\
\hline
\end{tabular}

Nontrivial solutions of the equation are defined by the characteristic equation

$$
\varepsilon^{4}-\varepsilon^{3} A_{1}+\varepsilon^{2} A_{2}-\varepsilon^{1} 3 A_{3}+A_{4}=0,
$$

which defines four eigenvalues $\varepsilon_{i}$. The scalar magnitudes $A_{i}$ are approximated by polynomials in the two-dimensional vector $\vec{\kappa}$. Symmetry operations of the small group with respect to the line $\mathrm{KH}$ (see Bradley-Cracknell [15, page 373]) leave their values invariant. Thus the $\vec{k} \cdot \vec{p}$ approximation only allows even powers of the norm of the $2 \mathrm{~d}$ vector $\vec{\kappa}$ with the important consequence that the Fermi surface remains unchanged under any rotation around the line $\mathrm{KH}$. The evaluation of the matrix elements $H_{i j}$ in the MAPW scheme is straightforward but quite tedious. We can avoid it by the fact that the characteristic equation (4) may be rewritten by use of the eigenvalues $\varepsilon_{i}$

$$
\left(\varepsilon-\varepsilon_{1}\right)\left(\varepsilon-\varepsilon_{2}\right)\left(\varepsilon-\varepsilon_{3}\right)\left(\varepsilon-\varepsilon_{4}\right)=0,
$$

which conversely allows to express the scalars $A_{i}$ by the four roots $\varepsilon_{i}$. Well known are the examples $A_{1}=\varepsilon_{1}+\varepsilon_{2}+\varepsilon_{3}+\varepsilon_{4}$ and 
$A_{4}=\varepsilon_{1} \varepsilon_{2} \varepsilon_{3} \varepsilon_{4}$. Analogous relations for $A_{2}$ and $A_{3}$ are easily derived.

Thus we can proceed in the following way.

(1) For a fixed value of $k_{\perp}$ and a set of values $|\vec{\kappa}|$ determine the roots $\varepsilon_{i}$ by solving the MAPW problem and evaluate the scalars $A_{i}$ by use of (4).

(2) In accord with the rotation invariance use the power expansions:

$$
\begin{aligned}
& A_{1}=\alpha_{1}+\beta_{1}|\vec{\kappa}|^{2}, \\
& A_{2} \approx \alpha_{2}+\beta_{2}|\vec{\kappa}|^{2}, \\
& A_{3}=\beta_{3}|\vec{\kappa}|^{4}, \\
& A_{4} \approx \beta_{4}|\vec{\kappa}|^{4} .
\end{aligned}
$$

for any value of $k_{\perp}$ and determine the coefficients $\alpha_{i}$ and $\beta_{i}$ by a least squares fit. Since the energy eigenvalues have been chosen relative to the energy value of the twofold degenerate state on the line $\mathrm{KH}$, the power expansion of $A_{3}$ and $A_{4}$ starts with $|\vec{\kappa}|^{4}$. The omitted terms in $A_{2}$ and $A_{4}$ are by a factor $|\vec{\kappa}|^{2}$ smaller than the included terms and are negligible for the modest values of $c$.

With this background, the strategy to find in $\vec{k}$ space the points of equal energy is established. The characteristic equation (4) combined with the power expansions equations (6) is an implicit equation which determines the radius $|\vec{\kappa}|$ of the circle connecting points of equal energy. As a consequence of the nonzero values $\beta_{3}$ and $\beta_{4}$ it is a quadratic equation defining either two different sheets or no sheet at all. Depending on the sign of the derivative $d|\vec{\kappa}|^{2} / d \varepsilon$, which can also be obtained from the characteristic equation, these circles run on a electron or hole sheet. Finally, the number of states $Z_{+}(\varepsilon)$ per unit volume with energies lower than $\varepsilon$ is obtained by integrating over the area of the circles with positive sign of $d|\vec{\kappa}|^{2} / d \varepsilon$ :

$$
Z_{+}(\varepsilon)= \pm \frac{2}{(2 \pi)^{3}} \int_{0}^{2 \pi / c} \pi|\vec{\kappa}|^{2} d k_{\perp} .
$$

This 1d integral is mostly restricted to subdomains where the integrand is defined. The analogous expression $Z_{-}(\varepsilon)$ counts the hole states with energies greater than $\varepsilon$.

The semimetallic behaviour of graphite requires that at least one electron and one hole-like sheet exist. In the case that the energy $E_{\mathrm{K}}$ is larger than $E_{\mathrm{H}}$, the hole-like surface originates from the point $K$, whereas the electron-like surface develops from the point $\mathrm{H}$. The exact compensation of the electron- and hole-like states defines the correct Fermi energy by the implicit equation

$$
Z_{+}\left(\varepsilon_{F}\right)+Z_{-}\left(\varepsilon_{F}\right)=0
$$

which is suitably solved near an estimated value $\varepsilon_{0}$

$$
\varepsilon_{F}=\varepsilon_{0}-\frac{Z_{+}\left(\epsilon_{0}\right)+Z_{-}\left(\epsilon_{0}\right)}{N_{+}\left(\epsilon_{0}\right)+N_{-}\left(\epsilon_{0}\right)} .
$$

Here $N_{ \pm}=d Z_{ \pm} / d \varepsilon$ is nothing else than the density of electron and hole-like states per unit volume. This strategy produces the value the Fermi energy with the same accuracy as the one-particle energies $E_{\vec{k}}$.

At this point the extraordinary small energy scale used in Figure 2 needs a special consideration since we cannot expect that the MAPW scheme guarantees this high accuracy even when more than 600 plane waves are chosen according to (2). Thus it may happen that with slightly different values $k_{\perp}$, distinctly different sets of plane waves are used with the consequence that the corresponding radii $\left|\vec{\kappa}\left(\varepsilon, k_{\perp}\right)\right|$ show distinctly visible jumps on the relevant length scale in $\vec{k}$ space. Such a jump may be seen in the case of $c=20.0$ Bohr near $k_{\perp}=0.4 \pi / c$. It allows to estimate the accuracy of the Bloch energies to be less than $0.25 \mathrm{mRy}$. Consequently the bands in the case $c=24.0$ Bohr show distinct fluctuations. To avoid them the bands displayed have been found by use of the same set of plane waves along the whole line.

The result of this refined analysis for different values of the interlayer distance $c$ is compiled in Table 2. It is remarkable that the number of electron or hole-like states $Z_{+}$is decay by three orders of magnitude when the interlayer distance is doubled. The density of states at the Fermi level $N_{ \pm}$shows a weaker decay, but the decrease of the metallicity is still pronounced.

Only for $c=12.6536$ Bohr a comparison with measurements is possible. Even our sophisticated treatment of the metallicity overestimates the number of conduction electrons and holes per $\mathrm{C}$ atom, which is defined by $V_{c}\left|Z_{ \pm}\left(\epsilon_{F}\right)\right| / 4$, by a factor of 4. McClure [13] found that his calculated carrier densities $2.4 \times 10^{-5}$ per atom for electrons and $1.8 \times 10^{-5}$ per atom for holes are in rough agreement with estimates derived from galvanomagnetic data.

Measurements of the heat capacity [22,23] give the value $N\left(\epsilon_{F}\right)=0.145$ states per Ry and cell. This discrepancy can certainly be ascribed to many body effects beyond the LDA approximation which are completely neglected in the present work. According to [5], the electron-electron correlation produces the main corrections. It is suspected that in graphite the phonon enhancement is negligible.

\section{Further Results}

5.1. The Fermi Surface. According to the preceding section the Fermi surfaces are rotationally symmetric around the line $\mathrm{KH}$ even in the case of the experimental value of $c$. This is in contradiction to a series of previous investigations $[1,2,5]$. With our choice of the parameter QMXSQ the electron-like surfaces are found to be located at the point $\mathrm{K}$, whereas the compensating hole-like states are located around the point $\mathrm{H}$ in the case $c=12.6536,16.0$ and 24.0 a.u. This is in accordance with previous investigations [24-26]. In the case $c=20.0$ a.u. the arrangement is reversed.

Figure 3 shows sections of a plane through the line $\mathrm{KH}$ with surfaces of equal energies magnified in radial direction that yield similarly shaped curves. The black lines describe electron-like states, and hole-like states are marked red. The Fermi surfaces around the plane КГМ have approximately the shape of extremely thin spindles. As a consequence of 
TABLE 2: Fermi energy, volume of elementary cell $V_{c}$, number of the states with energies below $Z_{-}$and above the Fermi energy $Z_{+}$, and density of electron and hole states per unit volume at the Fermi level $N_{ \pm}\left(\epsilon_{F}\right)$. All magnitudes are expressed in corresponding atomic units.

\begin{tabular}{lccccc}
\hline$c$ & $V_{c}$ & $\epsilon_{F}$ & $Z_{ \pm}\left(\epsilon_{F}\right)$ & $N_{+}\left(\epsilon_{F}\right)$ & $N_{-}\left(\epsilon_{F}\right)$ \\
\hline 12.6536 & 236.0474 & 1.295935 & $\pm 0.640110^{-5}$ & $0.146410^{-1}$ & $0.146410^{-1}$ \\
16.0 & 298.4730 & 1.00513582 & $\pm 0.126910^{-6}$ & $0.504110^{-2}$ & $0.337210^{-2}$ \\
20.0 & 373.0912 & 0.76237693 & $\pm 0.129510^{-7}$ & $0.887310^{-3}$ & $0.884110^{-3}$ \\
24.0 & 447.7094 & 0.59982141 & $\pm 0.156410^{-7}$ & $0.232410^{-3}$ & $0.122410^{-3}$ \\
\hline
\end{tabular}
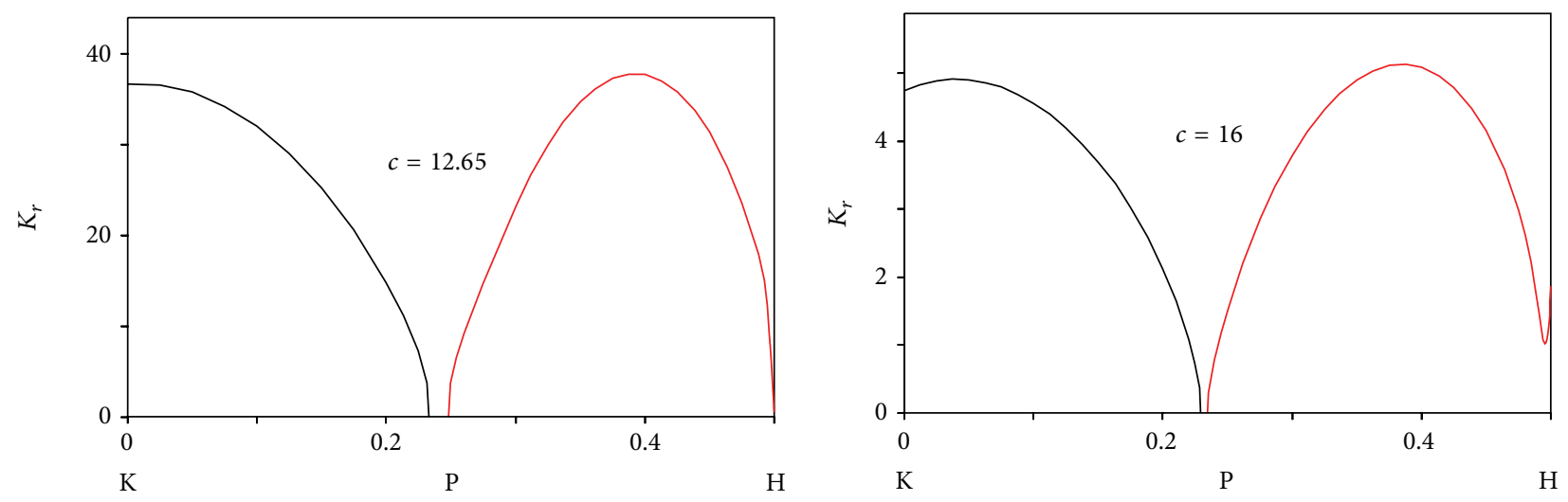

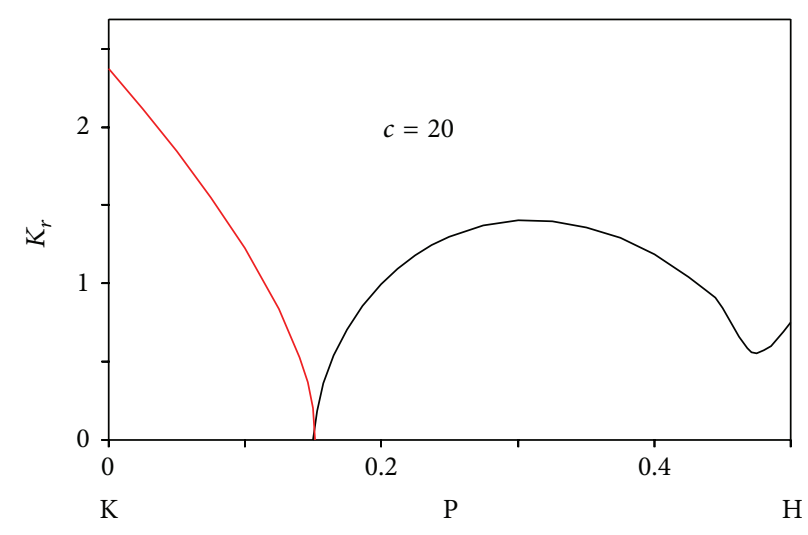

(a)

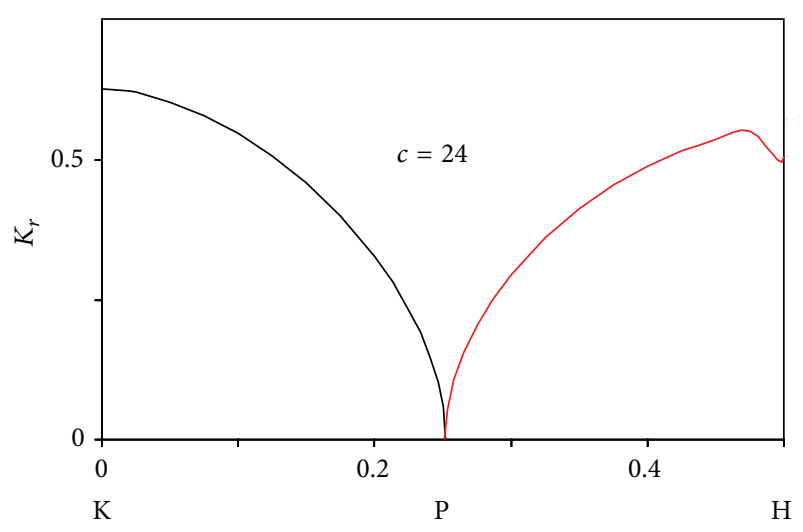

(b)

FiguRE 3: Intersections of planes through line $\mathrm{KH}$ with the surface of equal energies. Black line: electron states, red line: hole states. Dimensions in units of $2 \pi / a, 2 \pi / c$; respectively; however, the radii are enlarged by a factor of 10000 . Rotation around the line $\mathrm{KH}$ generates the corresponding Fermi surface.

the existence of a mirror plane, the operation $\sigma$ of the point group $D_{6 h}$, those around the plane HLA, are double spindles with minimal calibres near the mirror plane. Values of the extremal cross sections are listed in Table 3. Again they are decayed by 3 orders of magnitude with the doubling of the interlayer distance. They clearly demonstrate how the Fermi surfaces shrink to the line $\mathrm{HK}$ with increasing value of $c$, with the consequence of extremely small de-Haas-vanAlphen frequencies. The cyclotron masses $m_{c}$ as well as the inverse transport masses $m_{\text {trns }}^{-1}$ also listed in the table show a distinctly weaker decrease. The latter is defined by the sum in $\vec{k}$ space

$$
m_{\mathrm{trns}}^{-1}=\frac{1}{3 N} \operatorname{Spur} \sum_{n \vec{k}} \frac{\partial \epsilon_{n \vec{k}}}{\partial k_{\mu}} \frac{\partial \epsilon_{n \vec{k}}}{\partial k_{v}} \frac{\partial f}{\partial \epsilon}\left(\epsilon_{n \vec{k}}\right)
$$

where $N$ is the number of electron per cell. A highly simplified treatment of the dc conductivity based on the assumption of a relaxation time $\tau$ describing the scattering of electrons by lattice defects or phonons gives for an element of the dc tensor

$$
\sigma_{\mu \nu}=e^{2} \sum_{n \vec{k}} \tau_{n \vec{k}} \frac{\partial \epsilon_{n \vec{k}}}{\partial k_{\mu}} \frac{\partial \epsilon_{n \vec{k}}}{\partial k_{\nu}} \frac{\partial f}{\partial \epsilon}\left(\epsilon_{n \vec{k}}\right),
$$

which constitutes, as consequence of (10), nothing else than a generalised Drude formula. Thus the transport mass allows to estimate the magnitude of the $\mathrm{dc}$ conductivity. It also determines the plasma frequency $\omega_{\mathrm{pl}}^{2}=4 \pi e^{2} N m_{\text {trns }}^{-1} / \hbar$.

Measurements of the oscillatory magneto resistance (Shubnikov-de Haas Effect) of a high-quality graphite single crystal yield effective mass values in the basal plane of 0.039 
TABLE 3: Extremal areas, cyclotron masses, transport masses of electron (e) and hole (h) Fermi surfaces. The areas are in units of $(2 \pi / a)^{2}$, and the masses are multiples of the free electron mass.

\begin{tabular}{lccccrc}
\hline$c$ & $A_{\text {ext, } e}$ & $m_{c, e}$ & mass $_{\text {trns }, e}$ & $A_{\text {ext, } h}$ & $m_{c, h}$ & mass $_{\text {trns }, h}$ \\
\hline 12.6536 & $0.774210^{-4}$ & $0.119510^{-1}$ & $0.706910^{-2}$ & $0.446710^{-3}$ & $-0.102310^{-1}$ & $-0.872710^{-2}$ \\
16.0 & $0.129310^{-5}$ & $0.396510^{-3}$ & $0.355710^{-2}$ & $0.821110^{-4}$ & $-0.231610^{-1}$ & $-0.709210^{-2}$ \\
20.0 & $0.113210^{-6}$ & $0.505610^{-3}$ & $0.344210^{-3}$ & $0.177110^{-6}$ & $-0.901110^{-3}$ & $-0.344210^{-3}$ \\
24.0 & $0.249010^{-7}$ & $0.149110^{-3}$ & $0.181810^{-3}$ & $0.956510^{-8}$ & $-0.758910^{-4}$ & $-0.251910^{-3}$ \\
\hline
\end{tabular}

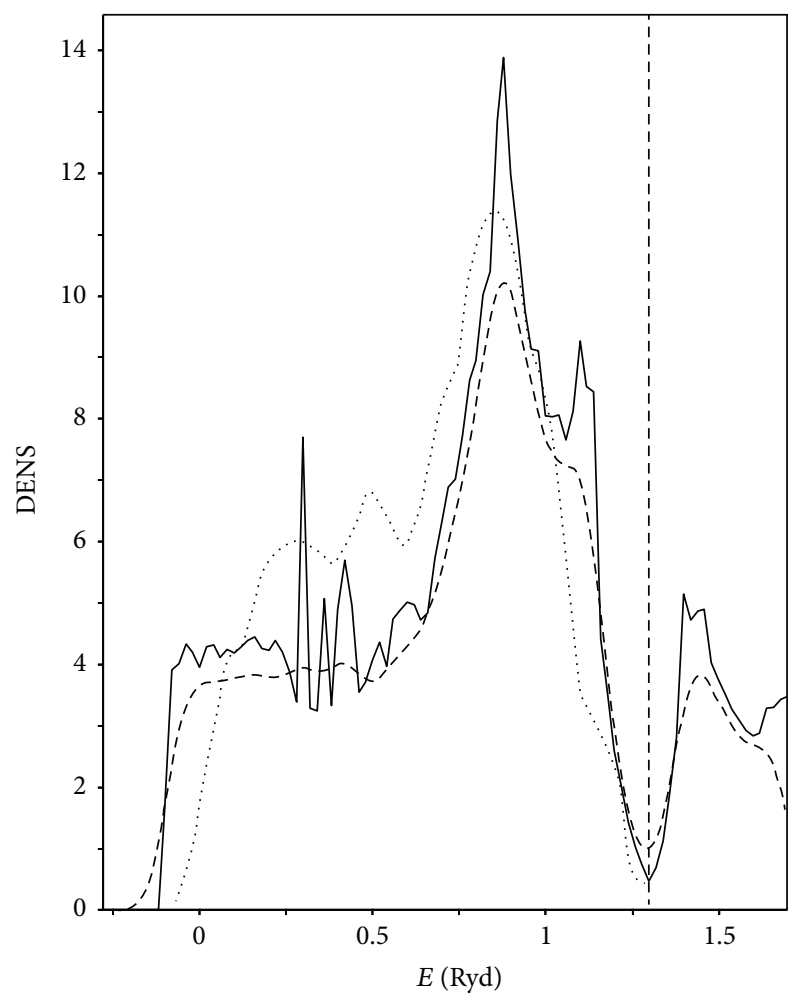

Figure 4: The density of states (DOS) for graphite. The full line shows the calculated DOS, while the broken line is obtained by convoluting with a Gaussian of FWHM $=0.05 \mathrm{Ry}^{-2}$. The dotted line shows the experimental X-ray photo emission spectra (XPS) [21].

free electron masses for electrons and 0.057 free electron masses for holes [27] which are significantly larger than theoretical values quoted in Table 3. The de-Haas-van-Alphen periods are 1.55 and $2.1 \times 10^{-5} \mathrm{G}^{-1}$, respectively, electrons at $\mathrm{K}$ and holes at $\mathrm{H}[28]$.

5.2. Density of States (DOS). The electronic density of states as function of the energy $N(E)$ has been calculated using the Gilat-Raubenheimer scheme [29] using $150 \vec{k}$ points in the Brillouin zone. For details see [30]. The typical step function in Figure 4 is due to the lack of dispersion along the line ML. The pronounced peak at $E \approx 0.85$ Ry can be associated with the highest occupied state at the point L. In accordance with the previous discussion we find a deep notch at the Fermi level. In a crude manner we took into account the correlation enhancement by folding $N(E)$ with a Gaussian of

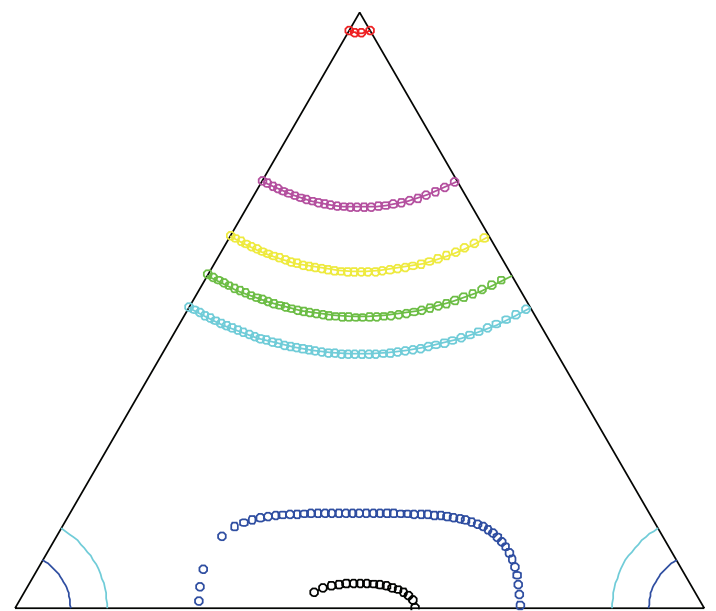

FIgURE 5: Charge-density contours for hexagonal graphite at the experimental value of the lattice constants, $a=4.6412 \mathrm{Bohr}, c=$ 12.6536 Bohr. The vector $\left(\vec{a}_{1}+2 \vec{a}_{2}\right) / 3$ points from the A-type atom, right-hand side corner to the B-type atom, left-hand side corner. The vector $\left(\vec{a}_{1}-\vec{a}_{2}\right) / 3$ points from the A-type atom to the centre of the hexagonal ring. The charge-density contours $\rho_{\mathrm{val}}=0.025,0.050$, $0.075,0.100,0.125,0.25$, and 0.28 electrons $\mathrm{Bohr}^{-3}$ are indicated by the colours red, bright red, yellow, green, bright blue, blue, and black, respectively.

width 0.1 Ry. This broadening results in a curve is compared favourably with the spectra obtained by X-ray photo emission spectroscopy [21].

5.3. Charge Density. Figure 5 shows the charge-density contours of the valence electrons in the fundamental triangle consisting of the two basis atoms in the lower corners and the centre of the hexagonal ring in the upper corner. The chargedensity $\rho_{\text {val }}$ assumes pronounced values near the two basis atoms; that is, $\rho_{\text {val }}=4.9568$ and 4.3869 electrons $\mathrm{Bohr}^{-3}$ at atoms of type A and type B, respectively, decreases rapidly and reaches minimal values near the line through the centre. Outside the circle which is the cut of the APW sphere with the plane: of the hexagonal ring, solely the plane wave part of the wave functions contributes to the charge density. Between the two basis atoms we find a secondary maximum with the value 0.28 electrons Bohr $^{-3}$ which explains the covalent bonds between the different atoms of the hexagonal ring. As consequence of the symmetry $D_{6 h}$ the contours around the centre of the hexagonal ring are almost spherically symmetric. These results qualitatively agree with previous 


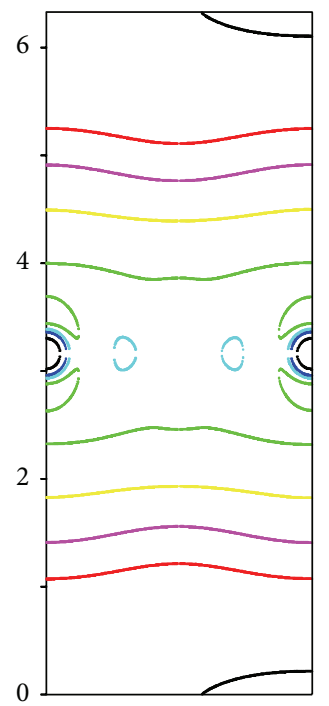

FIGURE 6: Charge-density contours for hexagonal graphite at the experimental value of the lattice constants in the fundamental quadrangle spanned by the vectors $\left(\vec{a}_{1}+2 \vec{a}_{2}\right) / 3$ pointing in the horizontal direction and the vector $\vec{a}_{3} / 2$ pointing in the vertical direction. In both directions the same scale, in Bohr, is used. The median of the quadrangle is the cut with the hexagonal ring. The charge-density contours $\rho_{\text {val }}=0.005,0,025,0.05,0.1,0.2,0.3,0.4$, and 0.8 electrons Bohr ${ }^{-3}$ are indicated by the colours black, red, light-red, yellow, green, bright-blue, black, respectively. Note that the vertical midline is not a cut with the reflection plane.

investigations [8]. The charge density in the plane spanned by the vectors $\left(\vec{a}_{1}-\vec{a}_{2}\right) / 3$ and $\vec{a}_{3} / 2$ is displayed in Figure 6 . The quasi two-dimensional behaviour of graphite is obvious from the charge-density distribution in the plane containing the hexagonal ring, since most of the charge density is found in the planes, with very little overlap between the different planes. Again the secondary maximum of the density distribution on the line connecting the atoms of type A and type B is quite pronounced. By comparing Figure 7 which also shows the charge-density contours in the fundamental quadrangle for the enlarged $c$ value $36.0 \mathrm{Bohr}$ with Figure 6 we see that the overlap and interaction between the different planes decrease substantially with growing values of $c$. Thus the results derived for the biggest value of $c$ well describe the charge-density distribution of graphene.

\section{Conclusions and Outlook}

With the careful precautions described perviously it is shown that the Fermi surface (FS) of crystalline graphite, even in the case of the measured interlayer distance, is essentially located around the line $\mathrm{KH}$ in the reciprocal space. It evolves from a twofold degenerate eigenstate on this line. In the light of the $\vec{k} \cdot \vec{p}$ approximation and the small extension of the FS in the plane perpendicular to the line $\mathrm{KH}$ it is evident that the angular decomposition of the Bloch functions defined on the Fermi surface consists mainly of a term depending on the $z$ coordinate which is especially located at the atoms of

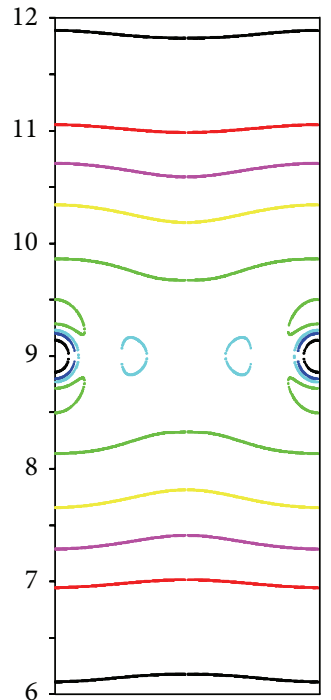

Figure 7: Charge-density contours for hexagonal graphite at the lattice constants $a=4.6411 \mathrm{Bohr}$ and $c=36.0 \mathrm{Bohr}$ in the fundamental quadrangle. To show the weak dependence on $c$ in the limit of large values the horizontal scale has been reduced by the factor 12.6526/36.0. For further details see Figure 6.

B type. Since approximately only half of the charge density is found to be within the APW spheres we have great doubts whether investigations based on the tight binding scheme or on the concept of localised Wannier functions give reliable results. The expansion of the interlayer distance $c$ favours the semimetallic property of graphite accompanied with a further shrinking of the Fermi surface, for example, for $c=36.0 a$ Bohr the FS dimensions are reduced by an order of magnitude. This insight will considerably simplify the evaluation of other ground state properties based on the band structure near the Fermi surface. For example in (11) we can assume the relaxation time $\tau_{n \vec{k}}$ on the Fermi surface to depend only on $k_{\perp}$. As the total charge density strongly is decayed outside the atomic layers the results derived for the biggest value of $c$ provide a good description for the case of graphene which is planned to be investigated in the near future.

\section{Acknowledgments}

Many thanks are to Dr. Herbert Stöhr and Dr. Reinhold Bader for helpful comments and the critical reading of the paper. The generous hospitality of Professor Jan von Delft and Professor U. Schollwöck is gratefully acknowledged.

\section{References}

[1] R. C. Tatar and S. Rabii, "Electronic properties of graphite: a unified theoretical study," Physical Review B, vol. 25, p. 4126, 1982.

[2] J. C. Charlier, X. Gonze, and J. P. Michenaud, "First-principles study of the electronic properties of graphite," Physical Review $B$, vol. 43, no. 6, pp. 4579-4589, 1991. 
[3] M. C. Schabel and J. L. Martins, "Energetics of interplanar binding in graphite," Physical Review B, vol. 46, p. 7185, 1992.

[4] J. Furthmüller, J. Hafner, and G. Kresse, " $A b$ initio calculation of the structural and electronic properties of carbon and boron nitride using ultrasoft pseudopotentials," Physical Review B, vol. 50, p. 15606, 1994.

[5] A. Grüneis, C. Attaccalite, L. Wirtz et al., "Tight-binding description of the quasiparticle dispersion of graphite and fewlayer graphene," Physical Review B, vol. 78, no. 20, p. 205425, 2008.

[6] H. J. F. Jansen and A. J. Freeman, "Structural and electronic properties of graphite via an all-electron total-energy local-density approach," Physical Review B, vol. 35, p. 8207, 1987.

[7] D. A. Fisher, R. M. Wentzcovitch, R. G. Carr, A. Continenza, and A. J. Freeman, "Graphitic interlayer states: a carbon K nearedge x-ray-absorption fine-structure study," Physical Review B, vol. 44, p. 1427, 1991.

[8] R. Ahuja, S. Auluck, J. Trygg, J. M. Wills, O. Eriksson, and B. Johansson, "Electronic structure of graphite: effect of hydrostatic pressure," Physical Review B, vol. 51, no. 8, pp. 4813-4819, 1995.

[9] J. C. Böttger, "Long-range magnetic order of the Er ions in $\mathrm{Er}_{2} \mathrm{Ba}_{4} \mathrm{Cu}_{7} \mathrm{O}_{14.92}$ ", Physical Review B, vol. 55, p. 11201, 1997.

[10] H. Bross, G. Bohn, G. Meister, W. Schubö, and H. Stöhr, "New version of the modified Augmented-Plane-Wave method," Physical Review B, vol. 2, p. 3098, 1970.

[11] H. Bross, "Electronic structure of the cubic compounds $\mathrm{ReGa}_{3}$ $(\mathrm{Re}=\mathrm{Er}, \mathrm{Tm}, \mathrm{Yb}$, and Lu)," Advances in Condensed Matter Physics, vol. 2011, Article ID 867074, 14 pages, 2011.

[12] P. R. Wallace, “The band theory of graphite," Physical Review, vol. 71, p. 622, 1947.

[13] J. W. McClure, "Band structure of graphite and de Haas-van Alphen effect," Physical Review, vol. 108, p. 612, 1957.

[14] J. C. Slonczewski and P. R. Weiss, "Band structure of graphite," Physical Review, vol. 109, no. 2, pp. 272-279, 1958.

[15] C. J. Bradley and A. P. Cracknell, The Mathematical Theory of Symmetry in Solids, section 1, Clarendon Press, Oxford, UK, 1972.

[16] H. Bross, "The local density approximation limit of the momentum density and the Compton profiles of Al," Journal of Physics: Condensed Matter, vol. 16, p. 7363, 2004.

[17] H. J. Monkhorst and J. D. Pack, "Special points for Brillouinzone integrations," Physical Review B, vol. 13, p. 5788, 1976.

[18] L. Hedin and B. I. Lundqvist, "Explicit local exchange-correlation potentials," Journal of Physics C, vol. 4, no. 14, article 022, pp. 2064-2083, 1971.

[19] S. H. Vosko, L. Wilk, and M. Nusair, "Accurate spin-dependent electron liquid correlation energies for local spin density calculations: a critical analysis," Canadian Journal of Physics, pp. 58-1200, 1980.

[20] P. Partoens and F. M. Peters, "From graphene to graphite: electronic structure around the K point," Physical Review B, vol. 74, Article ID 075404, 11 pages, 2006.

[21] A. Bianconi, S. B. M. Hagstrim, and R. Z. Bachkrach, "Photoemission studies of graphite high-energy conduction-band and valence-band states using soft-x-ray synchrotron radiation excitation," Physical Review B, vol. 16, p. 5543, 1977.

[22] P. H. Keesom and N. Pearlman, "Atomic heat of graphite between 1 and $20^{\circ} \mathrm{K}$," Physical Review, vol. 99, no. 4, pp. 1119-1124, 1955.
[23] J. C. Bowman and J. A. Krumhansl, "The low-temperature specific heat of graphite," Journal of Physics and Chemistry of Solids, vol. 6, p. 367, 1952.

[24] M. S. Dresselhaus and G. Dresselhaus, "Intercalation compounds of graphite," Advances in Physics, vol. 30, no. 2, pp. 139326, 1981.

[25] A. Grüneis, C. Attaccalite, T. Pichler et al., "Electron-electron correlation in graphite: a combined angle-resolved photoemission and first-principles study," Physical Review Letters, vol. 100, no. 3, Article ID 037601, 2008.

[26] S. Y. Zhou, G. H. Gweon, J. Graf et al., "First direct observation of Dirac fermions in graphite," Nature Physics, vol. 2, no. 9, pp. 595-599, 2006.

[27] D. E. Soule, J. W. McClure, and L. B. Smith, "Study of the Shubnikov-de Haas effect. Determination of the fermi surfaces in graphite," Physical Review, vol. 134, no. 2A, pp. A453-A470, 1964.

[28] J. A. Woolam, "Graphite carrier locations and quantum transport to $10 \mathrm{~T}$ (100 kG)," Physical Review B, vol. 3, p. 1148, 1971.

[29] G. Gilat and L. R. Raubenheimer, "Accurate numerical method of calculating frequency distribution functions in solids. II. Extension to hcp crystals," Physical Review, vol. 157, no. 3, pp. 586-599, 1967.

[30] H. Bross, "On the efficiency of different schemes for the evaluation of the density of states and related properties in solids," Physica Status Solidi, vol. 179, p. 429, 1993. 

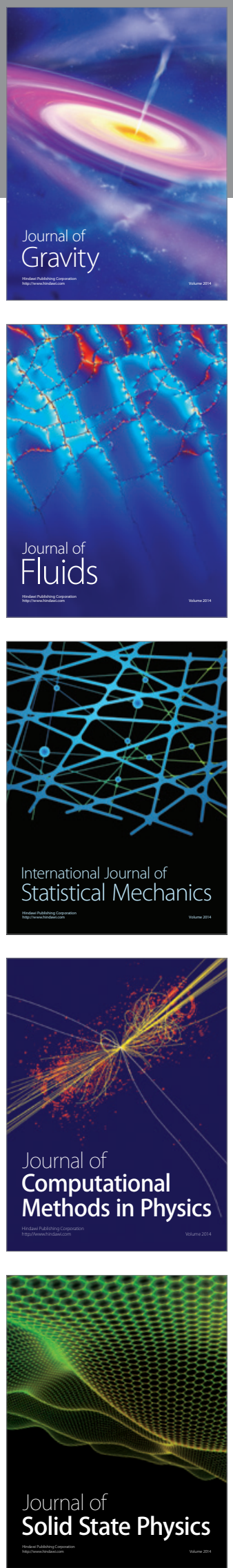

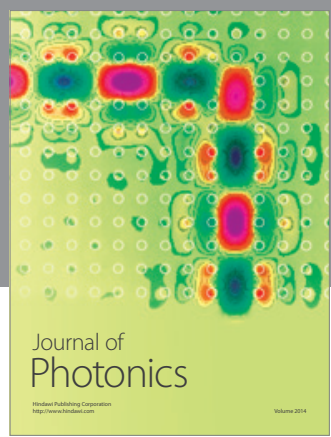

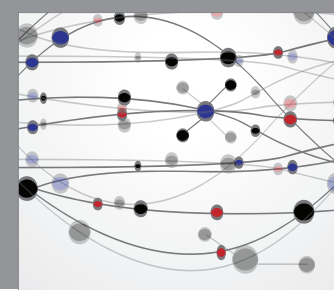

The Scientific World Journal

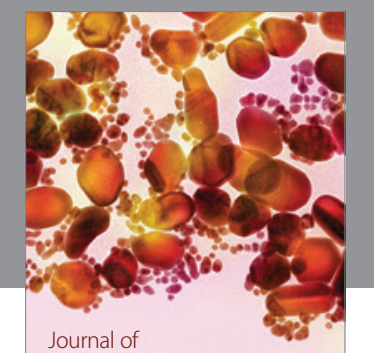

Soft Matter
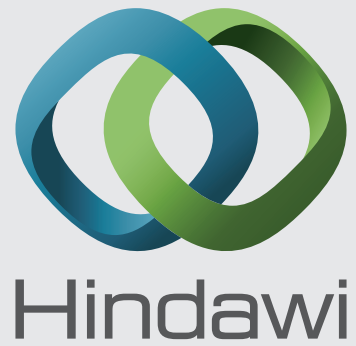

Submit your manuscripts at

http://www.hindawi.com
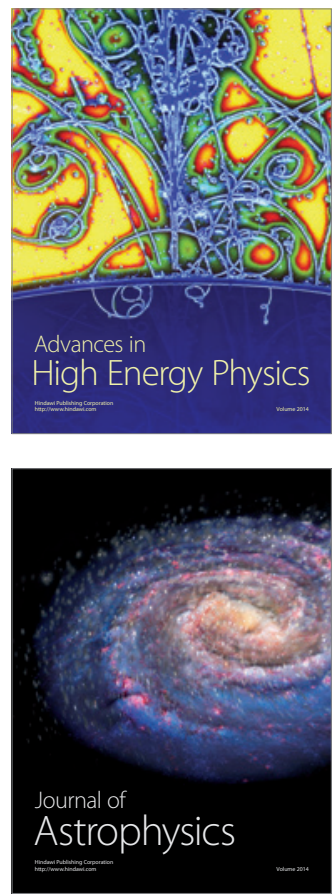
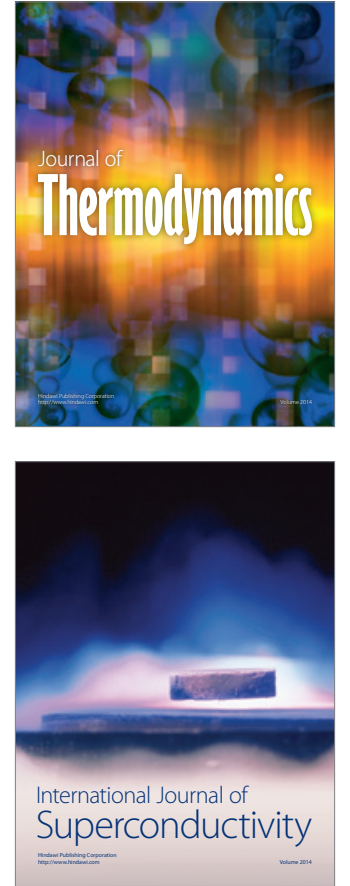
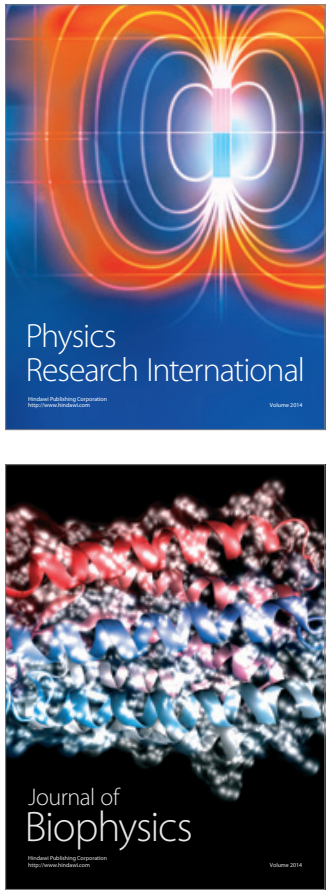
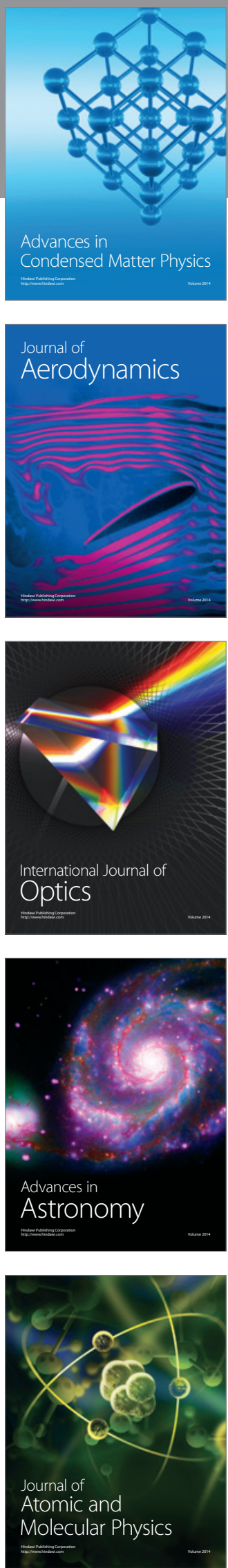